\title{
Clinical Effect of Traditional Chinese Medicine on Knee Pain: A Nationwide Population-Based Cohort Study
}

\author{
Yuan Chun $\mathrm{Lo}^{2}$, Nian Ze Hu${ }^{1}$, Po Hsu Chen², Ying Ling Chen², Mark C Hou ${ }^{2 *}$ and Tai Wei Kuo ${ }^{2}$ \\ ${ }^{1}$ Department of Information management, National Formosa University, Taiwan \\ ${ }^{2}$ Department of Chinese medicine, Changhua Christian Hospital, Taiwan
}

Submission: February 08, 2017; Published: January 25, 2018

*Corresponding author: Mark C Hou, No.135, Nanxiao St., Changhua City, Changhua County, Taiwan, Tel: +8864-7238595; Fax: +8864-7232942;

Email: dr.markhou@gmail.com

\begin{abstract}
Knee pain is a chronic progressive symptom that deteriorates the quality of life of elderly adults. Previous studies have indicated that knee pain can be ameliorated by acupuncture treatment. This study evaluated the clinical effect of the traditional Chinese medicine (TCM) on knee pain by analyzing the National Health Insurance Research Database (NHIRD) in Taiwan. We screened 240,000 randomly selected cases from the 2010 Longitudinal Health Insurance Database (LHID), a subset of the NHIRD, between 2001 and 2010. The inclusion criteria were patients who underwent total knee arthroplasty (TKA), were older than 45 years, and received a diagnosis for arthropathies (ICD-9 Codes 710 719). The patients were categorized according to their use of TCM. Cox regression models were employed to examine the hazard ratios (HRs) of the two groups. In addition, NodeXL was applied to analyze the core patterns according to the transformative comorbidities of the two groups of patients. Acupuncture can ameliorate knee pain; however, we found that TCM did not decrease the risk of TKA resulting from knee pain. Most patients with TKA had the comorbidity of hypertension.
\end{abstract}

Keywords: Knee pain; Total knee arthroplasty; Traditional chinese medicine

Abbreviations: TCM: Traditional Chinese Medicine; NHIRD: National Health Insurance Research Database; LHID: Longitudinal Health Insurance Database; TKA: Total Knee Arthroplasty; HRs: Hazard ratios

\section{Introduction}

Although advancements in medicine have lengthened human life expectancy, numerous issues associated with an aging society have accompanied this prolonged lifespan. For example, $28 \%$ of people older than 45 years in the United States have knee osteoarthritis, and this increased to $37 \%$ for people older than 65 years [1,2]. Knee osteoarthritis is a major factor associated with the inability to work, $[3,4]$ and knee pain from knee osteoarthritis is a chronic and common geriatric symptom that severely compromises the living quality of elderly adults [5]. In addition to being the largest joints in the human body, the knee joints are frequently used in various daily activities. Hence, knee pain typically impairs a person's movement. Knee osteoarthritis may be associated with repetitive external overload, which induces chronic intra-articular damage, leading to articular cartilage injury, bone sclerosis at the cartilage joints, formation of bone spurs and bone cysts, and thickening of the articular capsules and synovial bursas [6,7]. Currently, western medical interventions for knee osteoarthritis can be divided into conservative and invasive types. Conservative interventions include the use of medication, physiotherapy, and articular injection, whereas invasive interventions include arthroscopy, corrective osteotomy, and arthroplasty. Patients with severe latestage joint deformation often require total knee arthroplasty (TKA) followed by postoperative rehabilitation to restore walking function in the lower limbs [8,9]. Knee osteoarthritis is a progressive joint illness in which the joints deteriorate slowly and [10-12] continuously. The goal of treatment is to reduce pain, improve the range of motion, enhance joint function, and elevate living quality [9]. TKA is effective for alleviating joint pain in patients with late-stage knee-joint disorders, restoring knee joint function, and elevating life quality. TKA has a high success rate for treating knee disorders, and according to the Taiwan Central Health Insurance Bureau, TKA-associated medical expenditure was nearly NT $\$ 2.63$ billion in 2000, the second-highest hospitalization expenditure. Therefore, TKA is both critical treatment and common medical intervention for degenerative arthritis. 
While western medicine remains the most prominent form of treatment currently in use, alternative medicine and traditional Chinese medicine (TCM) have also begun to play a more critical role in medical systems. For example, the pain-alleviating effect of acupuncture has been demonstrated as a potentially effective treatment. In 1997, the National Institutes of Health (NIH) in the United States began to recommend acupuncture for reducing drug dosage [13]. For alleviating knee pain, using acupuncture for improving local muscle relaxation and promoting blood and qi circulation $[14,15]$. Subsequently, increasingly more patients and physicians have chosen acupuncture to improve the life quality of patients. The National Health Insurance Research Database (NHIRD), which contains the medical records of enrolees in Taiwan's National Health Insurance (NHI) program, is frequently used in research in Taiwan. Previous studies using the NHIRD have shown that TCM can reduce mortality in patients with chronic kidney disease, [16] and that acupuncture can decrease the reoccurrence of stroke [17]. In the present study, the NHIRD was used to sample patients with a diagnosed knee disorder who underwent knee arthroplasty and a retrospective cohort study was conducted for examining the clinical effectiveness of TCM interventions in alleviating knee joint pain.

\section{Materials and Methods}

\section{Research methods}

Data Sources: Data were obtained from the 2010 Longitudinal Health Insurance Database (LHID2010), which is maintained by the National Health Research Institutes. The medical records of 240,000 NHI beneficiaries for the 2001-2010 period were examined. The LHID2010 is a 10-year database containing data of randomly sampled beneficiaries of Taiwan's National Health Insurance program who are representative of the parent population. The data files include ambulatory care expenditures by visits (CD), details of ambulatory care orders (O0), and details of inpatient orders (DO). Ambulatory care expenditures by visits (CD) comprises sex (ID_SEX), personal ID (ID), date of birth code (ID_BIR), hospital department (FUNC_TYPE), hospital visiting date (FUNC_DATE), international statistical classification of diseases (ACODE_ICD9), and drug usage frequency (DRUG_FRE).

Data Analysis: The cause of knee-joint pain differs among age groups. Clinically, knee joint pain in elderly adults is commonly associated with degenerative arthritis, crystalinduced inflammatory arthropathy (gout, pseudogout...), popliteal cyst (Baker's cyst) and autoimmune rheumatic disease $[18,19]$. Because the present study investigated knee joint pain, Diagnosis Codes 710-719 for arthropathies and related disorders were selected as the key screening criteria of ACODE_ICD9 in the CD files.

Body weight is a key factor determining the severity of knee joint pain in knee osteoarthritis [20,21]. To ensure that the severity of knee osteoarthritis was as similar as possible between the experimental and control groups, body weight should be included in the screening criteria; however, the LHID2010 does not contain such information. Nevertheless, higher body weight is associated with a higher BMI, which is associated with increased probability of diabetes and cardiovascular disease [22-25]. Therefore, patients without cardiovascular disease or diabetes according to the diagnosis code were included in the exclusion criteria of this study.

Experimental group inclusion criteria: Patients who had received arthroplasty (Order Code 64164B) were selected from the DD data file. These patients were screened for arthropathies and related disorders (Diagnosis Codes 710-719) and were included only if they were 45 years or older based on ACODE_ ICD9 in the CD data file. Subsequently, the patients were rescreened to select those who had received a TCM intervention from the CD data file. The number, age, and sex of the patients were summarized. Finally, patients with incomplete data, those who underwent surgery before the initial arthropathy diagnosis, those without diagnosed hypertension or diabetes (based on the diagnosis codes of other diseases), and those with repeated data were excluded.

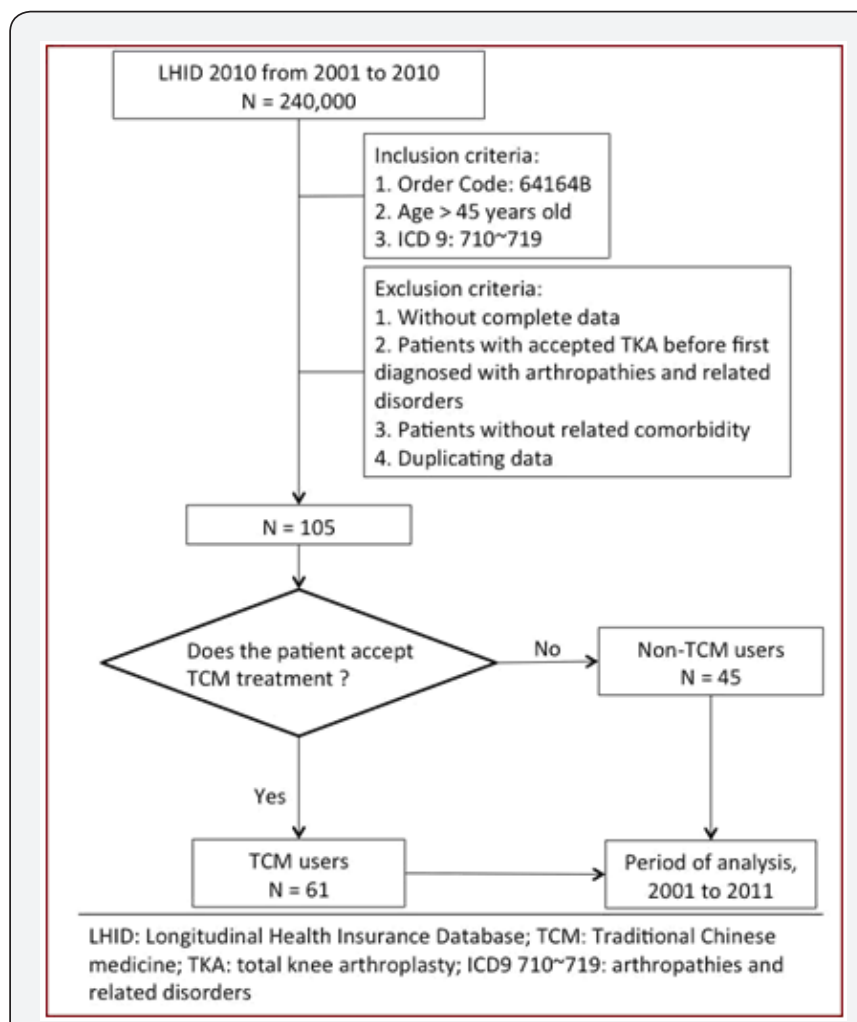

Figure 1: Flowchart of the study process.

Control group inclusion criteria: Patients who had received arthroplasty (Order Code 64164B) were selected from the DD data file. These patients were screened for arthropathies and related disorders (Diagnosis Codes 710-719) and were included only if they were 45 years or older based on ACODE_ICD9 in the $\mathrm{CD}$ data file. Subsequently, the patients were rescreened to select those who had received TCM intervention from the CD data 
file. The number, age and sex of the patients were summarized. Finally, patients with incomplete data, those who underwent surgery before the initial arthropathy diagnosis, those without diagnosed hypertension or diabetes (based on the diagnosis codes of other diseases), and those with repeated data were excluded (Figure 1).

\section{Statistical methods}

To calculate the postarthroplasty hazard ratio (HR) between those receiving both the TCM intervention and western medical intervention and those receiving western medical intervention alone, the Cox regression survival analysis was performed to determine whether the experimental and control groups demonstrated differences in sex, age, and the length of time between arthropathy diagnosis and arthroplasty.

NodeXL (http://nodexl.codeplex.com/), an open-source network analysis and visualization package for Microsoft Excel, is useful for analyzing social network relationships and identifying opinion leaders in specific virtual communities [26,27]. NodeXL was used in the present study to analyze the comorbidities in the experimental and control groups. In the LHID2010, each patient is assigned up to four diagnosis codes; however, because graphs generated using NodeXL have only two vertices on their edges, the diagnosis codes were transformed before processing. The first field was defined as follows: according to the four diagnosis codes, patients with hypertension alone were classified as Type 1 ; those with diabetes alone were classified as Type 2; and those with both hypertension and diabetes were classified as Type 3 . For the second field, patients with diagnosed conditions other than hypertension and diabetes were included. Subsequently, NodeXL network analysis was performed using the transformed data to explore the network centrality. SPSS Version 19.0 was used for the data analysis with the $\alpha$ level set to 0.05 ; thus, results were considered statistically significant when $\mathrm{p}<0.05$. This study was approved by the institutional review board of the study hospital (IRB N: 141002).

\section{Results}

From the LHID2010, this study obtained 870 patients satisfying the inclusion criteria. After the exclusion criteria were applied, the remaining 106 patients were divided into the experimental group $(n=61)$ and control group $(n=45)$ depending on whether they had received a TCM intervention.

The experimental group contained 17 men (28\%) and 44 women $(72 \%)$ (age mean, $69.65 \pm 7.17$ y) who had received treatment through both standard and TCM interventions. The average duration between their initial arthropathy diagnosis and arthroplasty was 2.97 years. The control group comprised 10 men $(22 \%)$ and 35 women (78\%) (age mean, $69.9 \pm 8.32$ y) who received a standard intervention only. The average duration between their initial arthropathy diagnosis and arthroplasty was 3.03 years (Table 1 ).
Table 1: Basic data of the experimental and control groups. No significant difference in gender or age was observed between the two groups.

\begin{tabular}{|c|c|c|c|c|}
\hline & \multicolumn{2}{|c|}{ Study Group(N=61) } & \multicolumn{2}{c|}{ Control Group(N=45) } \\
\hline Gender(n) & Male & Female & Male & Female \\
\hline & $17(28 \%)$ & $44(72 \%)$ & $10(22 \%)$ & $35(78 \%)$ \\
\hline Age & & $69.65 \pm 7.17$ & & $69.9 \pm 8.32$ \\
\hline $\begin{array}{c}\text { Time to } \\
\text { TKA (year) }\end{array}$ & & 2.97 & & 3.03 \\
\hline
\end{tabular}

Regarding the hazard ratio for patients receiving arthroplasty to treat knee joint pain, the male: female ratio was 1.019:1.000 (95.0\% CI, 0.648-1.604), and the ratio between the experimental and control groups was 1.044:1.000 (95.0\% CI, 0.705-1.547) (Table 2).

Table 2: Cox proportional HR by group and sex.

\begin{tabular}{|c|c|c|}
\hline & $\begin{array}{c}\text { Cox Proportional } \\
\text { Hazard Ratio }\end{array}$ & CI \\
\hline $\begin{array}{c}\text { Study gr. to control } \\
\text { gr. }\end{array}$ & 1.044 & 0.705 to 1.547 \\
\hline Male to female & 1.019 & $0.648-1.604$ \\
\hline
\end{tabular}

CI: 95\% confidence interval

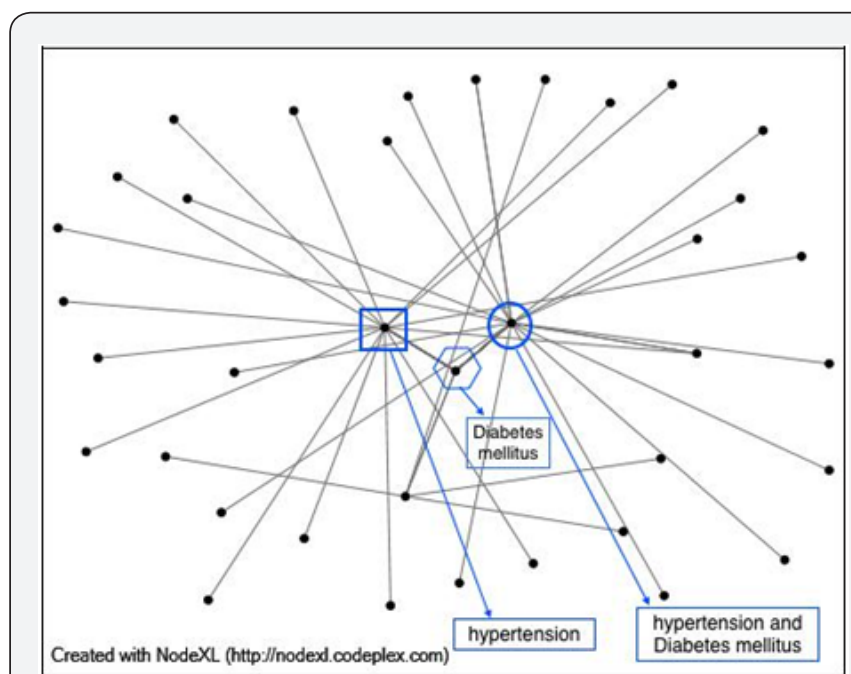

Figure 2: Fruchterman-Reingold graph for the comorbidities in the experimental group. Hypertension (sepure) and both hypertention and diabetes (circle) have centrality tendencies in the network.

Fruchterman-Reingold graphs were generated using NodeXL. In the graph for the experimental group (Figure 2), the square indicates the patients with hypertension and the circle represents the patients with both diabetes and hypertension. Both the square and circle show a centrality tendency in the network. Regarding graph for the control group (Figure 3), only the square shows a centrality tendency. Therefore, the NodeXL revealed that hypertension exhibited centrality in the sample comorbidity network. In other words, patients with knee joint pain who received an arthroplasty were more likely to have hypertension as well. 


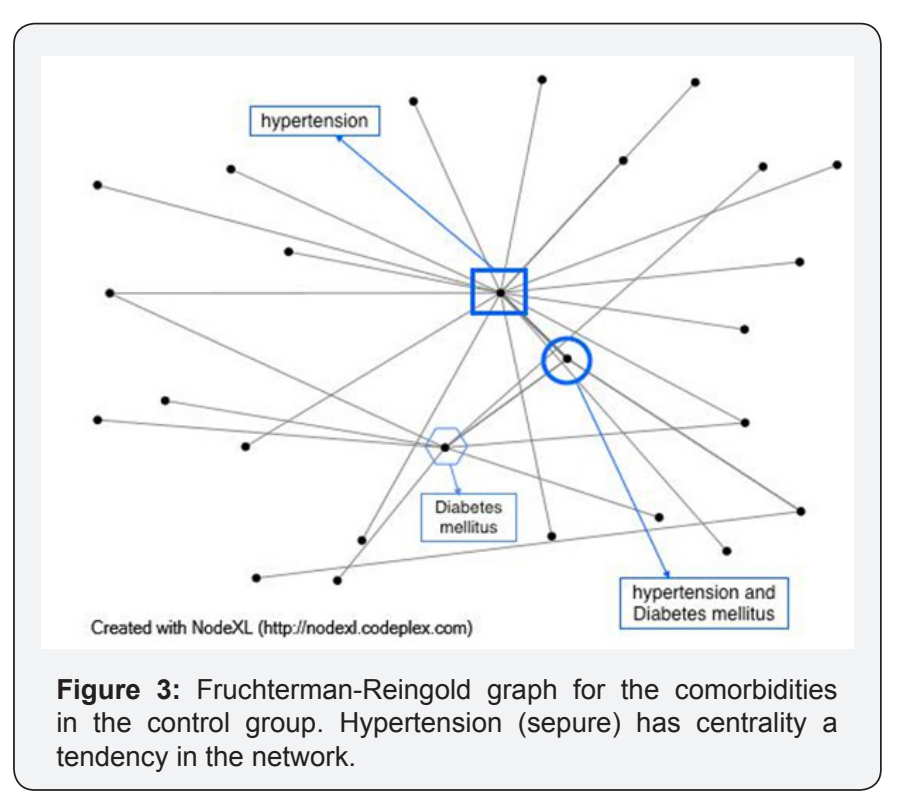

\section{Discussion}

The NHIRD contains the medical records of all people living in Taiwan who are enrolled in the NHI program, as well as information on the alternative complementary therapies they have received. The data examined in this study were from the LHID2010, which contains medical claims data representative of the general population in Taiwan. The inclusion criteria were patients who underwent TKA, older than 45 years, and received a diagnosis for arthropathies. And the exclusion criteria were patients with incomplete data, those who underwent surgery before the initial arthropathy diagnosis, those without diagnosed with related comorbidities, and those with repeated data. There was big difference between the initial and final cases. That was because only 140 cases met our criteria only 140 . Although researchers in Germany have already published clinical studies examining the effect of acupuncture on ameliorating knee joint pain $[10,12,28]$ their studies did not explore whether acupuncture can delay the need for TKA. The present study used real-world pattern data as well as a long follow-up period to verify the efficacy of acupuncture on TKA. Nonetheless, one limitation of a study involving the use of database is that the level of severity of the disease could not be determined. Although high body weight is a key factor of the severity of knee joint pain in degenerative arthritis, [20,21] the LHID2010 does not contain any BMI-related information. However, because a high BMI is associated with an increased probability of diabetes and cardiovascular disease, [22,29] patients without cardiovascular disease or diabetes were excluded on the basis of the diagnosis codes of other related diseases to ensure that the experimental and control groups were comparable. Due to pain is a subjective symptom, and is not proportional to the disease severity. And because the decision to undergo an arthroplasty was determined by the physician and the patient, and no standard is available for determining whether an arthroplasty is necessary, predicting the severity was difficult and evaluations of disease severity might vary.
According to the theory of TCM, knee osteoarthritis is a disease resulting from invasion of wind, cold, and dampness. There are many methods about treatment with TCM of knee osteoarthritis, including acupuncture, moxibustion, Chinese herbs, herbal patch, qigong, massage therapy etc. It can increase Qi flowing and blood circulating after TCM treatment and can relieve knee pain and swelling inducing by osteoarthritis. The previous systematic review study searched for studies in PubMed that were performed between 1965 and 2013, and retrieved studies were subjected to reference screening. The most commonly using acupoints were ST35, ST36, ST40, SP6, SP9, SP10, GB34, GB39, KI6, Ex-LE 2 and Ex-LE5; and the every time of treatment was about 20 30 minutes. Moreover, De Qi sensation, a feeling that indicates effective needling, was being enhanced [14]. Due to the data examined in this study from the LHID2010, one of limitations is that we could not know the acupoints and the treatment time in experimental group. But we could ensure the operator of acupuncture was TCM doctors, who had accepted the completed acupuncture training.

Western medical approaches for treating knee joint pain can be categorized as conservative and invasive, [8] the effectiveness of which may vary from person to person. Alternative therapies used in different countries and TCM have become crucial in medical systems worldwide [30]. In Taiwan, the preference for and usage rate of TCM are higher than those in many other countries [31] and there is clinical evidence showing that acupuncture is effective in alleviating various types of pain $[10,12,28]$. Therefore, a TCM intervention for ameliorating knee joint pain was investigated in the present study. In addition to age and sex, related comorbidities were also included in the inclusion criteria to ensure that the experimental and control groups show no significant difference in their profiles and level of disease severity. Nonetheless, the TCM interventions exhibited statistically nonsignificant effect on arthroplasty-related risks. This study inferred that the aforementioned results were attributed to patient lifestyle (e.g., job type and preferred physical activities) not being considered in the present study. In addition, many patients in Taiwan pay for acupunture without insurance; consequently, the NHIRD does not contain information on such treatments. If these two factors were accounted for in the statistical analysis, the results might have differed. Accordingly, these two factors should be investigated in future studies.

The cause of knee joint pain differs among age groups. Clinically, knee joint pain in elderly adults is commonly associated with degenerative arthritis, crystal-induced inflammatory arthropathy (gout, pseudogout...), popliteal cyst (Baker's cyst) and autoimmune rheumatic disease [18]. Therefore, initially, this study focused on diagnosis code ACODE_ICD9 715.63 when investigating knee joint pain. However, using this diagnosis code yielded an extremely small sample size. Consequently, Code 715.63 was replaced with Codes 710-719 (arthropathies and related disorders) to expand the coverage. Nevertheless, this study found that clinical physicians typically preferred using diagnosis code 715.36 (osteoarthrosis, localized, primary or 
secondary lower leg unspecified); therefore, when conducting database studies, researchers should investigate the routine clinical procedure and practice.

With technological advancements and the prevalence of the Internet, social network analysis has been adopted more frequently in recent studies. In contrast to conventional analysis methods, social network analysis considers each person or event as a node, between which a line is plotted. When the nodes are connected, a network structure is formed, which can more accurately reflect a social network of people and events in comparison with other methods. For example, a network structure of Facebook members could be built by connecting people according to their interpersonal relationships. Subsequently, the intersection demonstrating the highest level of centrality in the network can be used to infer the opinion leader of this group. In recent years, social network analysis approaches have been used frequently in medical research $[32,33]$. For example, Yen et al. employed NodeXL to analyze the network of TCM prescriptions for treating chronic sinusitis; Chen et al. [34] employed NodeXL to analyze the network of TCM for treating dysmenorrhea [33]. In the present study, NodeXL analysis was applied in examining comorbidity, and hypertension was intuitively identified as the point of centrality for comorbidity on the Fruchterman-Reingold graph. NodeXL was selected for this study because it is easy to operate; moreover, this open-source software can be used with Microsoft Excel. NodeXL also revealed that hypertension exhibited the strongest clustering effect, which was inferred to be attributable to the associated increase in blood pressure reducing the degree of sensitivity to pain $[35,36]$. Therefore, knee joint pain might be more severe than it is in reality, thus increasing the probability of receiving arthroplasty.

\section{Conclusions}

Acupuncture can ameliorate knee pain; however, we found that TCM did not postpone the duration for patient with knee pain to undergo TKA. Most patients with TKA had the comorbidity of hypertension. We hope there are more related studies in the future.

\section{Conflict of Interest}

The authors have no conflict of interests.

\section{References}

1. Lawrence RC, Felson DT, Helmick CG, Arnold LM, Choi H, et al. (2008) Estimates of the prevalence of arthritis and other rheumatic conditions in the United States. Part II. Arthritis Rheum 58(1): 26-35.

2. Jordan JM, Helmick CG, Renner JB, Luta G, Dragomir AD, et al. (2007) Prevalence of knee symptoms and radiographic and symptomatic knee osteoarthritis in African Americans and Caucasians: the Johnston County Osteoarthritis Project. J Rheumatol 34(1): 172-180.

3. Juni P, Reichenbach S, Dieppe P (2006) Osteoarthritis: rational approach to treating the individual. Best practice \& research. Best Pract Res Clin Rheumatol 20(4): 721-740.

4. Peat G, McCarney R, Croft P (2001) Knee pain and osteoarthritis in older adults: a review of community burden and current use of primary health care. Ann Rheum Dis 60(2): 91-97.

5. Kittelson AJ, Stevens LJE, Schmiege SJ (2015) Determination of pain phenotypes in knee osteoarthritis: A latent class analysis using data from the osteoarthritis initiative study. Arthritis Care Res 68(5): 612620 .

6. McAlindon T, Dieppe P (1989) Osteoarthritis: definitions and criteria. Ann Rheum Dis 48(7): 531-532.

7. Felson DT (1988) Epidemiology of hip and knee osteoarthritis. Epidemiol Rev 10: 1-28.

8. Hauk L (2014) Treatment of knee osteoarthritis: a clinical practice guideline from the AAOS. Am Fam Physician 89(11): 918-920.

9. Chou CL, Lo YT, Liu FW, Haung LL, Chen JJ (2012) The therapeutic effects of intra-articular hyaluronic acid on the physical functioning of patients with knee osteoarthritis: A preliminary report. Taiwan Journal of Physical Medicine and Rehabilitation. 40(2): 77-83.

10. Haake M, Muller HH, Schade BC, Basler HD, Schäfer H, et al. (2007) German acupuncture trials (GERAC) for chronic low back pain: randomized, multicenter, blinded, parallel-group trial with 3 groups. Arch Intern Med 167(17): 1892-1898.

11. Witt C, Brinkhaus B, Jena S, Linde K, Streng A, et al. (2005) Acupuncture in patients with osteoarthritis of the knee: a randomised trial. Lancet 366(9480): 136-143.

12. Molsberger AF, Schneider T, Gotthardt H, Drabik A (2010) German randomized acupuncture trial for chronic shoulder pain (GRASP)-a pragmatic, controlled, patient-blinded, multi-centre trial in an outpatient care environment. Pain 151(1): 146-154.

13. (1998) NIH Consensus Conference. Acupuncture. JAMA 280(17): 15181524.

14. Hou PW, Fu PK, Hsu HC, Hsieh CL (2015) Traditional chinese medicine in patients with osteoarthritis of the knee. J Tradit Complement Med 5(4): 182-196.

15. Tsuchiya M, Sato EF, Inoue M, Asada A (2007) Acupuncture enhances generation of nitric oxide and increases local circulation. Anesth Analg 104(2): 301-307.

16. Hsieh CF, Huang SL, Chen CL, Chen WT, Chang HC, et al. (2014) Nonaristolochic acid prescribed chinese herbal medicines and the risk of mortality in patients with chronic kidney disease: results from a population-based follow-up study. BMJ open 4(2): e004033.

17. Shih CC, Liao CC, Sun MF, Su YC, Wen CP, et al. (2015) A retrospective cohort study comparing stroke recurrence rate in ischemic stroke patients with and without acupuncture treatment. Medicine 94(39): e1572.

18. Calmbach WL, Hutchens M (2003) Evaluation of patients presenting with knee pain: Part II. Differential diagnosis. Am Fam Physician 68(5): 917-922.

19. Carr AJ, Robertsson O, Graves S, Andrew JP, Nigel KA, et al. (2012) Knee replacement. Lancet 379(9823): 1331-1340.

20. Felson DT, Goggins J, Niu J, Zhang Y, Hunter DJ (2004) The effect of body weight on progression of knee osteoarthritis is dependent on alignment. Arthritis Rheum 50(12): 3904-3909.

21. Creamer P, Lethbridge CM, Hochberg MC (2000) Factors associated with functional impairment in symptomatic knee osteoarthritis. Rheumatology (Oxford) 39(5): 490-496.

22. Wang S, Majumdar SR, Padwal R (2015) BMI, annual blood pressure measurements, and mortality in patients with obesity and hypertension: a retrospective cohort study. Blood press monit 20(1): 32-38. 
23. Ko GT, Chan JC, Cockram CS, Woo J (1999) Prediction of hypertension, diabetes, dyslipidaemia or albuminuria using simple anthropometric indexes in Hong Kong Chinese. International journal of obesity and related metabolic disorders. Int J Obes Relat Metab Disord 23(11): 1136-1142.

24. (2000) Obesity: preventing and managing the global epidemic. Report of a WHO consultation (WHO technical report series 894): 252.

25. Fernandes RA, Zanesco A (2015) Early sport practice is related to lower prevalence of cardiovascular and metabolic outcomes in adults independently of overweight and current physical activity. Medicina 51(6): 336-342

26. Derrible S (2012) Network centrality of metro systems. PloS one 7(7): e40575.

27. Fruchterman TMJ, Reingold EM (1991) Graph drawing by forcedirected placement. Softw Pract Exper 21(11): 1129-1164.

28. Bowing G, Zhou J, Endres HG, Coeytaux RR, Diener HC, et al. (2010) Differences in Chinese diagnoses for migraine and tension-type headache: an analysis of the German acupuncture trials (GERAC) for headache. Cephalalgia 30(2): 224-232.

29. WHO (2004) Appropriate body-mass index for Asian populations and its implications for policy and intervention strategies. Lancet 363(9403): 157-163.

30. Klein SD, Torchetti L, Frei EM, Wolf U (2015) Usage of complementary medicine in Switzerland: results of the swiss health survey 2012 and development since 2007. PloS one 10(10): e0141985.

31. Rau SP (2007) Study on Utilization of Traditional Chinese Trauma tologic Manipulation and Rehabilitation Medicine in Taiwan.

32. Chen HY, Lin YH, Hu S, Yang SH, Chen JL, et al. (2015) Identifying Chinese herbal medicine network for eczema: implications from a nationwide prescription database. Evidence-based complementary and alternative medicine 2015(2015): 347164.

33. Chen HY, Lin YH, Su IH, Chen YC, Yang SH, et al. (2014) Investigation on Chinese herbal medicine for primary dysmenorrhea: implication from a nationwide prescription database in Taiwan. Complement Ther Med 22(1): 116-125.

34. Yen HR, Sun MF, Lin CL, Sung FC, Wang CC, et al. (2015) Adjunctive traditional Chinese medicine therapy for patients with chronic rhinosinusitis: a population-based study. Int Forum Allergy Rhinol 5(3): 240-246.

35. Guasti L, Gaudio G, Zanotta D, Grimoldi P, Petrozzino MR, et al. (1999) Relationship between a genetic predisposition to hypertension, blood pressure levels and pain sensitivity. Pain 82(3): 311-317.

36. Bae YH, Shin JS, Lee J, Meriong K, Ki Byung P, et al. (2015) Association between hypertension and the prevalence of low back pain and osteoarthritis in Koreans: A cross-sectional study. PloS one 10(9): e0138790.

\section{Your next submission with Juniper Publishers will reach you the below assets}

- Quality Editorial service

- Swift Peer Review

- Reprints availability

- E-prints Service

- Manuscript Podcast for convenient understanding

- Global attainment for your research

- Manuscript accessibility in different formats

( Pdf, E-pub, Full Text, Audio)

- Unceasing customer service

Track the below URL for one-step submission https://juniperpublishers.com/online-submission.php 